\title{
Phosphoric Acid Mediated Glycosylation and Alcohol-Chirality Recognition
}

\section{Key words}

Brønsted acid glycosylation alcohols
Selected examples:<smiles>OC1CCCCC1P</smiles>

$( \pm)$
$0 \%$ yield $(\beta, S)$<smiles>CC[C@@H](C)O</smiles>

$( \pm)$ $17 \%$ yield $(\beta, S)$

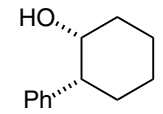

$( \pm)$

$87 \%$ yield $(\beta, R)$ $0 \%$ yield $(\beta, S)$<smiles>C[C@@H](O)C[O+]SP</smiles>

$( \pm)$ $8 \%$ yield $(\beta, S)$
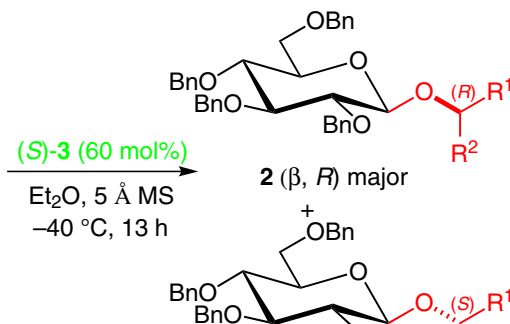

$2(\beta, R)$ major

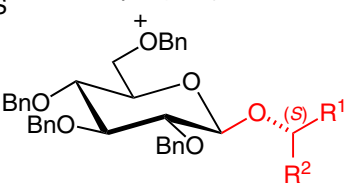

$2(\beta, S)$ minor

7 examples

$80-92 \%$ yield

$R / S$ from 5:1 to exclusively $R$

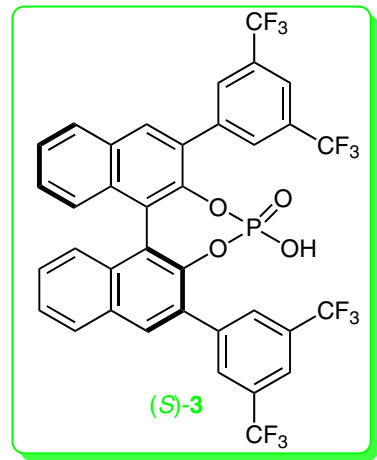

Reaction pathway:

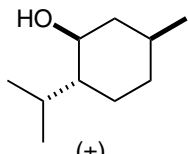

$67 \%$ yield $(\beta, R)$ $13 \%$ yield $(\beta, S)$

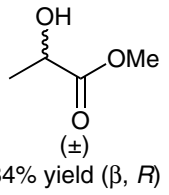
$0 \%$ yield $(\beta, S)$

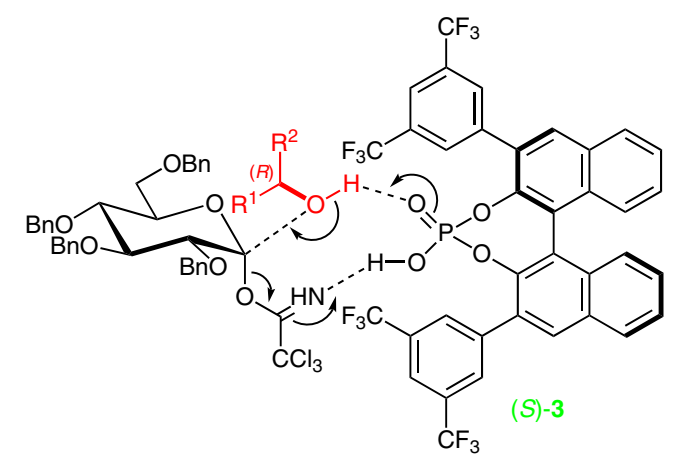

Significance: Toshima and co-workers report a highly $\beta$-selective glycosylation of $\alpha$-trichloroacetimidates $\mathbf{1} \alpha$ with various secondary alcohols. The diastereoselectivity is moderate to excellent, and the reaction is mediated by the phosphoric acid (S)-3. According to mechanistic studies, the exclusive $\beta$-selectivities are obtained through a (S)-3mediated $S_{N} 2$ reaction pathway. The methodology was also applied to the total synthesis of a natural flavan glycoside using a racemic aglycone.
Comment: Glycosylation is an important synthetic method to construct sugar moiety containing compounds. Here, the authors report a novel Brønsted acid mediated glycosylation, and a kinetic resolution of secondary alcohols occurs during the process at the same time. This methodology provides a straightforward way for the synthesis of sugar-derived products with high stereoselectivity. 